Address for Correspondence: Dr. Muhammed Mubarak, Department of Histopathology, Sindh Institute of Urology and Transplantation, Karachi 74200, Pakistan.

E-mail: drmubaraksiut@yahoo.com

\begin{tabular}{|l|}
\hline Access this article online \\
Website: \\
www.intern-med.com \\
\hline DOI: \\
10.4103/2224-4018.147747 \\
\hline Quick Response Code: \\
\hline
\end{tabular}

\title{
Unusual presentation of nonalcoholic steatohepatitis-related cirrhosis in a patient with celiac disease and microscopic colitis
}

\author{
Muhammed Manzoor, Rajesh K. Wadhwa, Zaigham Abbas, Syed Mujahid Hasan, \\ Nasir Hasan Luck, Muhammed Mubarak ${ }^{1}$ \\ Departments of Hepatogastroentrology and 'Histopathology, Sindh Institute of Urology and Transplantation, \\ Karachi, Pakistan
}

\section{ABSTRACT}

Nonalcoholic steatohepatitis (NASH) is defined as the presence of hepatic steatosis and inflammation with hepatocyte injury (ballooning) with or without fibrosis. NASH is often a "silent" liver disease. Estimated prevalence of NASH ranges from 3\% to $5 \%$ in different studies. The prevalence of NASH-related cirrhosis in the general population is not known. Herein, we report a case of a young female presented with NASH-related cirrhosis in the setting of poorly controlled celiac disease (CD) and microscopic colitis. A variety of liver abnormalities have been observed in patients with $C D$, but this unique constellation of the gut and liver pathologies has not been reported previously.

Key words: Celiac disease, cirrhosis, fatty change, liver, microscopic colitis

\section{INTRODUCTION}

Nonalcoholic steatohepatitis (NASH) is defined as the presence of hepatic steatosis and inflammation with hepatocyte injury (ballooning) with or without fibrosis. NASH is often a "silent" liver disease. Estimated prevalence of NASH ranges from 3\% to 5\% in different studies. ${ }^{[1]}$ Patients with NASH can develop progressive liver disease, and cirrhosis. The prevalence of NASH-related cirrhosis in the general population is not known. ${ }^{[1]}$ The estimated prevalence of nonalcoholic fatty liver disease (NAFLD) in Pakistan is $18 \%{ }^{[2]}$ The clinical spectrum starts with simple hepatic steatosis, characterized by triglyceride accumulation in hepatocytes and ending up in the progressive form of NASH, which may even be associated with the development of cirrhosis or even hepatocellular carcinoma. ${ }^{[3,4]}$

Generally, nonalcoholic fatty liver disease/ NASH occurs in obese patients. However, in few cases, NASH occurs in thin lean patients, such as those with celiac disease
(CD). A close association between the gut and liver pathology, has previously been shown for inflammatory bowel disease. ${ }^{[5]}$ An association between $C D$ and liver injury (chronic active hepatitis) has previously been described as well as its close relation to steatosis and steatohepatitis. ${ }^{[6]} \mathrm{CD}$ has even been described as being associated with acute, severe acute and chronic hepatitis. ${ }^{[7-9]}$

Approximately, half of all untreated CD patients show elevated transaminases. ${ }^{[9-11]}$ In approximately $40 \%$ of all adults with $\mathrm{CD}$, increased serum transaminase levels are found at diagnosis ${ }^{[12]}$ and such elevations may be the only abnormality in cases of "occult" CD. ${ }^{[13]}$ In fact, in a study by Bardella et al., 9.3\% of cases of unexplained chronic hypertransaminasemia were ultimately diagnosed as $\mathrm{CD} \cdot{ }^{[12]}$

Herein, we report a case of a young female diagnosed as NASH-related cirrhosis with seronegative $\mathrm{CD}$ and microscopic colitis (MC). NASH-related cirrhosis in the setting of CD has not been observed in 
previous studies or case reports. Occurrence of NASHrelated cirrhosis in $\mathrm{CD}$ may have a true correlation rather than a coincidence this constellation of gastrointestinal pathologies is extremely rare.

\section{CASE REPORT}

A 25-year-old thin, lean, unmarried, female presented with the complaint of abdominal distension, which was gradual and progressive in nature, associated with mild swelling of feet, and no history of abdominal pain or fever. She had a history of $10 \mathrm{~kg}$ weight loss over 4 months. She has a past history of spinal tuberculosis (TB) in 2004 and took antituberculous treatment for 1-year. Her grandfather had pulmonary TB and father died of hepatitis $C$ virus (HCV)-related chronic liver disease (CLD). There was no history of diabetes, hypertension and alcohol intake. On examination, her body mass index (BMI) was 20.5. Abdomen was distended with fullness of flanks; fluid thrill was elicited with audible gut sounds. The rest of systemic examination was unremarkable.

Laboratory investigations showed low serum sodium $(126 \mathrm{mEq} / \mathrm{L})$ with normal liver function tests, fasting blood sugar and fasting lipid profile. Ultrasonography and computed tomography abdomen showed marked fatty liver infiltration, omental thickening and caking, but no abdominal lymphadenopathy. Spleen and portal vein were normal. Small right sided pleural effusion and a large amount of ascites were noted. Ascitic fluid analysis showed high serum ascitic albumin gradient, low protein, with total leucocyte count of $180 / \mathrm{mm}^{3}$ with $53 \%$ lymphocytes and $46 \%$ neutrophils. Thyroid profile was normal. Echocardiography showed ejection fraction of $55 \%$ with normal left ventricular function. Hepatitis B surface antigen and anti $\mathrm{HCV}$ and antinuclear antibody were negative. Urinary copper was normal and serum ceruloplasmin was reduced to $0.17 \mathrm{~g} / \mathrm{L}$ (normal: $0.2-0.6 \mathrm{~g} / \mathrm{L}$ ). There was no Kayser-Fleischer ring on slit lamp examination. On esophagogastroduodenoscopy, no varices were seen, but there was severe fissuring with decreased height of duodenal folds. Duodenal biopsy showed villous atrophy, flattening of surface, crypt hyperplasia consistent with Marsh Type III lesion [Figure 1a]. Her human leucocyte antigen typing was DQ2 and DQ8, whereas antitissue transglutaminase antibodies were negative. Colonoscopy and ileoscopy were normal. Colonic biopsy showed features of MC with foci of lymphocytic cryptitis [Figure 1b]. Diagnostic laparoscopy showed omental portion adherent to the abdominal wall in the right iliac fossa, yellowish macronodular liver suggestive of fatty changes. Liver biopsy revealed severe steatosis with mild to moderate expansion of portal tracts with lymphocytic infiltrate and focal ballooning of hepatocytes. Interface hepatitis with bridging fibrosis with occasional nodules and foci of lobulitis also noted. All these features suggested NASH with a fibrosis score of 5/6 [Figure $1 \mathrm{c}$ and d].

Gluten free diet was instituted and she was given pentoxifyllin and Vitamin E. She has shown improvement in her clinical condition and laboratory results and is on regular follow-up.

\section{DISCUSSION}

Obesity is a common and a well-known risk factor for NAFLD, with the prevalence of the latter being reported as high as $90 \%$ in severe obesity. ${ }^{[14]}$ There is also a very high prevalence $(69 \%)$ of NAFLD in Type II diabetes mellitus. ${ }^{[14]}$ High serum triglycerides and low HDL are also common associations of NAFLD/NASH. On the other hand, rapid weight loss and severe starvation is also associated with NAFLD. ${ }^{[15]}$

The diagnosis of NAFLD/NASH needs exclusion of other possible causes of CLD. Liver biopsy is the gold standard for the diagnosis. In our case, the patient presented with uncontrolled CD with rapid weight loss of $10 \mathrm{~kg}$ over a period of 4 months, which may be a contributing factor for NASH progression. Fat mobilization with rapid weight loss may accelerate the inflammatory process and this was evident in liver biopsy, which revealed severe steatosis with mild to moderate expansion of portal tracts with lymphocytic infiltrate and focal ballooning of hepatocytes. All these features were suggestive of NASH with fibrosis score of $5 / 6$.

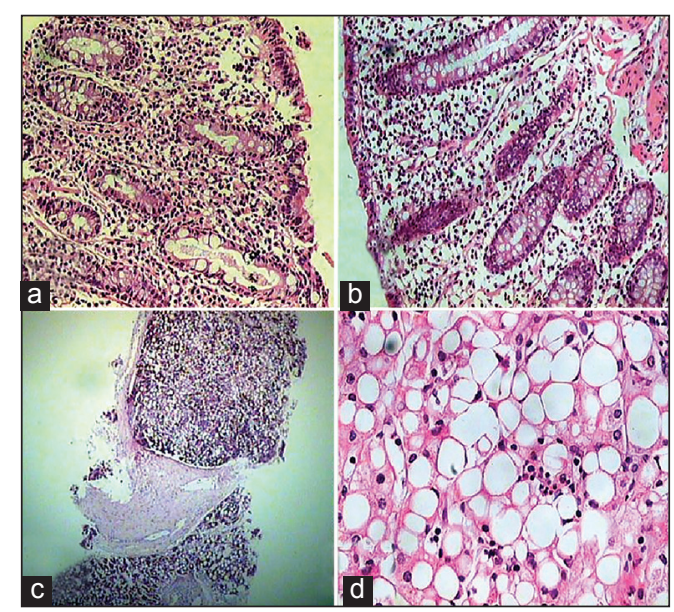

Figure 1: (a) High-power photomicrograph showing complete villous atrophy, with flattening of the surface and crypt hyperplasia. These findings are consistent with Marsh Type III lesion (H and $E, \times 400)$. (b) High-power photomicrograph showing numerous lymphocytes infiltrating the crypt and surface epithelium of the colonic mucosa. There is no crypt distortion or neutrophilic infiltration $(H$ and $E, \times 400)$. (c) Low-power photomicrograph showing portal and periportal fibrosis with partial nodule formation consistent with stage $5 / 6$ fibrosis of liver (PAS, $\times 100)$. (d) Highpower photomicrograph showing severe fatty change and focal inflammatory cell infiltration consistent with steatohepatitis $(H$ and $E, \times 400)$ 
Colonic biopsy revealed features of MC, which has a strong association with $\mathrm{CD}{ }^{[16]}$ This may cause persistent diarrhea despite strict adherence to gluten free diet.

The variety of liver abnormalities in $\mathrm{CD}$ is particularly broad. Abnormalities of liver function are one of the manifold extraintestinal manifestations of $\mathrm{CD}$. CD has been found in $2-3 \%$ of patients with primary sclerosing cholangitis, 3-6\% with autoimmune hepatitis and $3-7 \%$ of patients with primary biliary cirrhosis. ${ }^{[16]}$

Nonalcoholic fatty liver disease is common in the general population but its occurrence in $\mathrm{CD}$ has been observed rarely. One of the study reported the overall prevalence of CD of $2.2 \%$ in patients with NAFLD, and it was $5.8 \%$ in patients with BMI value $<27 \mathrm{~kg} / \mathrm{m}^{2} \cdot{ }^{[16]}$ However, to the best of our knowledge, the presence of NASH-related cirrhosis in the setting of $\mathrm{CD}$ and $\mathrm{MC}$ has not been observed in previous studies or case reports. The concurrence of NASH-related cirrhosis in CD may be a true association rather than a coincidence that needs to be validated by more studies or case reports.

Treatment options for NASH include lifestyle changes, control of diabetes, hyperlipidemia and reduction of cardiovascular risk. Moderately calorie restricted diet, moderate exercise and drugs targeting insulin resistance are the mainstay treatment options. Antifibrotic and antioxidant agents and liver transplantation are also treatment options. The primary treatment of $\mathrm{CD}$ is the removal of gluten from the diet. In addition to gluten restriction, we have given antioxidants pentoxifyllin and Vitamin $\mathrm{E}$ to our patient and managed her for complications of liver cirrhosis.

\section{CONCLUSION}

Colonoscopy with biopsy should be performed in patients with refractory CD to rule out MC. In addition, patients with hepatic steatosis on imaging and signs and symptoms attributable to liver disease or abnormal liver biochemistry should be evaluated for NAFLD/NASH and related complications.

\section{REFERENCES}

1. Vernon G, Baranova A, Younossi ZM. Systematic review: The epidemiology and natural history of non-alcoholic fatty liver disease and non-alcoholic steatohepatitis in adults. Aliment Pharmacol Ther 2011;34:274-85.

2. LaBrecque D, Abbas Z, Anania F, Ferenci F, Khan AG, Goh K-L, et al. Nonalcoholic fatty liver disease and nonalcoholic steatohepatitis, World Gastroenterology Organization Global Guidelines, June 2012. Available from: http://www.worldgastroenterology.org/assets/export/ userfiles/2012_NASHandNAFLD_Final_long.pdf. [Last accessed on 2012 August 01].

3. Ertle J, Dechêne A, Sowa JP, Penndorf V, Herzer K, Kaiser G, et al. Non-alcoholic fatty liver disease progresses to hepatocellular carcinoma in the absence of apparent cirrhosis. Int J Cancer 2011;128:2436-43.

4. Canbay A, Gieseler RK, Gores GJ, Gerken G. The relationship between apoptosis and non-alcoholic fatty liver disease: An evolutionary cornerstone turned pathogenic. Z Gastroenterol 2005;43:211-7.

5. Eade MN, Cooke WT, Williams JA. Liver disease in Crohn's disease. A study of 100 consecutive patients. Scand J Gastroenterol 1971;6:199-204.

6. Vajro P, Fontanella A, Mayer M, De Vincenzo A, Terracciano LM, D'Armiento M, et al. Elevated serum aminotransferase activity as an early manifestation of gluten-sensitive enteropathy. J Pediatr 1993;122:416-9.

7. Kaukinen K, Halme L, Collin P, Färkkilä M, Mäki M, Vehmanen P, et al. Celiac disease in patients with severe liver disease: Gluten-free diet may reverse hepatic failure. Gastroenterology 2002;122:881-8.

8. Abdo A, Meddings J, Swain M. Liver abnormalities in celiac disease. Clin Gastroenterol Hepatol 2004;2:107-12.

9. Volta U. Liver dysfunction in celiac disease. Minerva Med 2008;99:619-29.

10. Volta U. Pathogenesis and clinical significance of liver injury in celiac disease. Clin Rev Allergy Immunol 2009;36:62-70.

11. Bardella MT, Fraquelli M, Quatrini M, Molteni N, Bianchi P, Conte D. Prevalence of hypertransaminasemia in adult celiac patients and effect of gluten-free diet. Hepatology 1995;22:833-6.

12. Bardella MT, Vecchi M, Conte D, Del Ninno E, Fraquelli M, Pacchetti S, et al. Chronic unexplained hypertransaminasemia may be caused by occult celiac disease. Hepatology 1999;29:654-7.

13. Chalasani N, Younossi Z, Lavine JE, Diehl AM, Brunt EM, Cusi K, et al. The diagnosis and management of non-alcoholic fatty liver disease: Practice Guideline by the American Association for the Study of Liver Diseases, American College of Gastroenterology, and the American Gastroenterological Association. Hepatology 2012;55:2005-23.

14. Rafiq N, Younossi ZM. Effects of weight loss on nonalcoholic fatty liver disease. Semin Liver Dis 2008;28:427-33.

15. Rahimi AR, Daryani NE, Ghofrani H, Taher M, Pashaei MR, Abdollahzade S, et al. The prevalence of celiac disease among patients with non-alcoholic fatty liver disease in Iran. Turk J Gastroenterol 2011;22:300-4.

16. Stewart M, Andrews CN, Urbanski S, Beck PL, Storr M. The association of coeliac disease and microscopic colitis: A large population-based study. Aliment Pharmacol Ther 2011;33:1340-9.

How to cite this article: Manzoor M, Wadhwa RK, Abbas Z, Hasan SM, Luck NH, Mubarak M. Unusual presentation of nonalcoholic steatohepatitis-related cirrhosis in a patient with celiac disease and microscopic colitis. J Transl Intern Med 2014;2:172-4.

Source of Support: Nil, Conflict of Interest: None declared 